\title{
Cardio-oncology Training in the COVID-19 Era
}

Stephanie Feldman, $M D^{1}$

Jennifer Liu, MD ${ }^{1,2}$

Richard Steingart, $M D^{1,2}$

Dipti Gupta, MD, MPH ${ }^{1,2, *}$

\author{
Address \\ ${ }^{*}, 1$ Cardiology Division, Department of Medicine, Memorial Sloan-Kettering Cancer \\ Center, 1275 York Ave, New York, NY, 10065, USA \\ Email: guptad@mskcc.org \\ ${ }^{2}$ Weill Cornell Medical College, New York, NY, USA
}

Published online: 7 June 2021

(C) The Author(s), under exclusive licence to Springer Science+Business Media, LLC, part of Springer Nature 2021

This article is part of the Topical Collection on Cardio-oncology

Keywords Cardio-oncology · Fellowship training · Medical education · Cardiotoxicity · COVID-19

\section{Opinion Statement}

Cardio-oncology is a field dedicated to the prevention, diagnosis, and management of cardiovascular disease in cancer patients before, during, and after cancer therapy. It is an emerging field with limited opportunities for structured education and training. In the year 2021, we cannot define the requirements of cardio-oncology training without acknowledging the impact of the global coronavirus disease 19 (COVID-19) pandemic. While this pandemic poses significant health risks to patients with cancer and cardiovascular disease as well as the providers who care for them, it also allows novel opportunities for the nascent field of cardio-oncology to readily adapt. In this article, we detail how the COVID-19 pandemic has impacted all aspects of cardio-oncology training, how programs and trainees can adapt to these challenges, and how lessons learned from the COVID-19 era can continue to positively impact cardio-oncology training for the foreseeable future.

\section{Introduction}

Over the past 30 years, there have been major advances in the early detection and targeted treatment of cancer. This has led to improved survival rates, with the projected number of cancer survivors expected to increase to over 22 million by 2024 [1]. Cancer survivors include a rapidly growing population with short- and long-term cardiovascular toxicities of cancer and cancer-related therapy. Cardio-oncology is a burgeoning field that focuses on the prevention and management of cardiovascular disease at all stages of cancer therapy and 
survivorship. Several groups have put forth standards to be considered competent in cardio-oncology along with suggestions on how to develop a cardio-oncology fellowship program $[2,3]$. Due to the growing number of patients who would benefit from dedicated cardiooncology care, it is prudent to train family practitioners, primary care physicians, advance practice providers, cardiologists, and oncologists in pertinent aspects of primary and secondary prevention of cardiovascular disease in cancer patients. Guidelines for this are well delineated in recommendations recently published by the American College of Cardiology (ACC) Cardio-

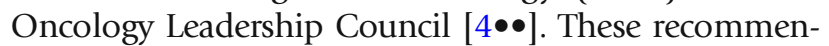
dations are proposed utilizing the standardized COCATS (Core Cardiology Training Symposium) structure, which is currently used for cardiology training programs. This framework allows trainees to gain experience during their general residency or fellowship (COCATS level I, II) or during a dedicated advanced cardio-oncology fellowship training year (COCATS level III).

In the year 2021, with an ongoing global pandemic that has impacted more than 26 million US citizens and resulted in over 535,000 US deaths, it is imperative to consider how the coronavirus disease 19 (COVID-19) has impacted the cardio-oncology patient population, the practice of cardio-oncology, as well as the training of future cardio-oncologists [5]. The virus is thought to spread through respiratory droplets by individuals who are symptomatic and by those who are infected without active symptoms [6]. Due to this mechanism of spread and concern for rapid community transmission, many outpatient visits and elective clinical procedures were cancelled or delayed at the beginning of the pandemic. As it became clear, the pandemic would not be a short-lived phenomenon, clinicians turned to telemedicine to provide patient care while minimizing exposure risk to vulnerable patients and to healthcare providers.

Some patients with cancer, cardiovascular disease, or both cancer and cardiovascular disease are at higher risk of severe COVID-19 and death [7-9]. As demonstrated by studies performed at our institution, cancer patients at increased risk for severe COVID-19 include those over 65 years old, recently treated with immune checkpoint inhibitors, active hematologic malignancy, active lung cancer, and clonal hematopoiesis [10-12]. On the other hand, recent chemotherapy, recent major surgery, or previous hematopoietic stem cell transplantation (allogeneic or autologous) or chimeric antigen receptor (CAR) T cell therapy were not associated with worse COVID-19 outcomes in cancer patients [10,13]. This data is an additional tool utilized to triage and manage highrisk cancer and cardio-oncology patients at our institution.

Given the increased risk of severe COVID-19 in many cardio-oncology patients, it has been necessary to think creatively about how to minimize patient exposures to this highly contagious virus while simultaneously providing necessary medical care. During the initial months of the outbreak and during periods of peak transmission, this meant transitioning to telemedicine in both the inpatient and outpatient settings and considering decreased frequency of outpatient cardiotoxicity surveillance testing for asymptomatic patients. Similarly, to minimize the risk of disease transmission to healthcare workers, the traditional framework for medical education has required a dramatic makeover. As the Centers for Disease Control and Prevention (CDC) guidelines recommend avoidance of gatherings of more than ten people and to keep six feet between individuals, inperson didactic sessions, imaging sessions, and large national conferences have been cancelled or replaced with virtual options [6]. At the time of this article, there are currently two mRNA vaccines that have received US Food and Drug Administration Emergency Use Authorization for the prevention of COVID-19 [14, 15]. Due to limited supply, health care workers, residents of longterm care facilities, and other essential workers have been the first to receive the vaccine. Patients over 65 and those with comorbid conditions such as cardiovascular disease and cancer will be the next population eligible for vaccination [16]. With adequate personal protective equipment (PPE), vaccinated providers and patients, cardio-oncology patients and providers may return to more in-person visits and regularly scheduled surveillance testing. However, the pandemic is unlikely to end soon and will continue to change traditional modes of clinical care and medical education for the foreseeable future.

Cardio-oncology training consists of four major components: inpatient and outpatient clinical experience, didactic instruction, and research. The COVID-19 pandemic has impacted all of these areas, serving as a catalyst for what will likely be lasting change in the field of cardio-oncology (Fig. 1). 


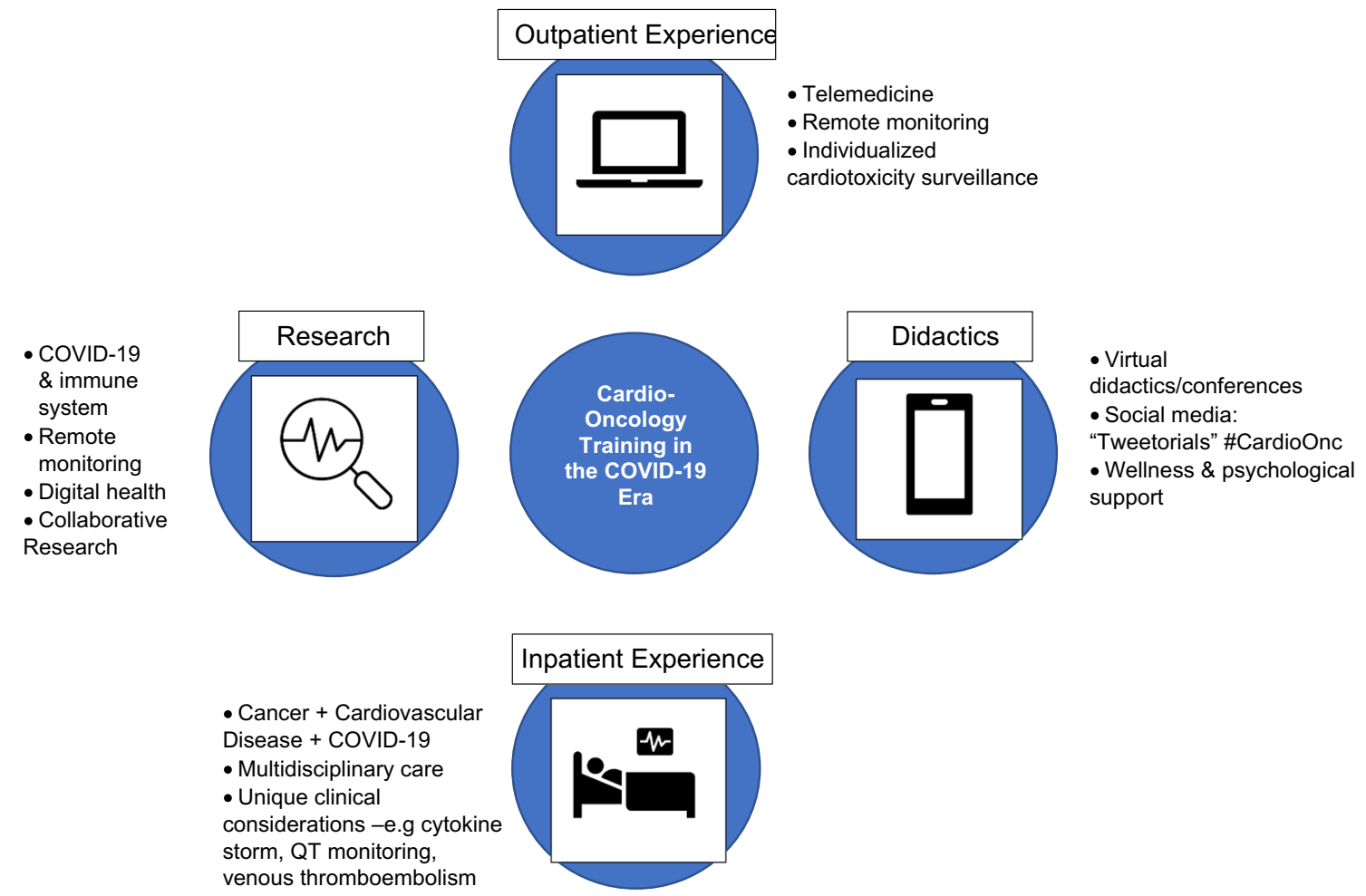

Fig. 1. Core components of cardio-oncology training in the COVID-19 era.

\section{Outpatient clinical experience}

A key component to cardio-oncology training is clinical experience, in both the outpatient and inpatient settings. The ACC Cardio Oncology Leadership Council propose that COCATS level II and III trainees have at least 20 and 40 half-day clinic sessions, respectively $[4 \bullet \bullet]$. In the outpatient setting, telemedicine or telehealth is an effective means of reducing unnecessary patient and provider exposure. Telehealth includes chart review, telephone visit, and video visit between provider and patient using secure HIPAA (Health Insurance Portability and Accountability Act)-compliant platforms such as Doximity (San Francisco, $\mathrm{CA}$ ) or Cisco Jabber (Denver, CO). Each of these modalities has strengths and weaknesses, with the major strengths including increased access to specialized cardio-oncology expertise and allowing for timely assessment. Limitations include lower reimbursement rates (e-consultations in particular), physicians must be licensed in the state where patients are located, the inability to perform a thorough physical exam, multiple technical glitches, and difficulty establishing rapport through a screen $[17 \bullet \bullet]$. At MSK, cardio-oncology fellows participate in an average of four clinic sessions per month with two different faculty preceptors. During the height of the pandemic in New York, the majority of outpatient visits were performed via telemedicine with attending cardio- 
oncologists pre-screening clinics and arranging in-person evaluation for select clinical questions, e.g., heart failure assessment or complicated pre-operative cardiac risk evaluation. For telemedicine visits, the cardio-oncology fellow and attending have a chance to review the clinic schedule prior to each session, allowing the fellow the opportunity to ask any content-specific questions prior to the patient encounter. The telemedicine visit is performed via three-way video or telephone call with the fellow, attending, and patient together. Telemedicine assessment can be supplemented by at-home medical equipment and digital health technology $[17 \bullet \bullet, 18]$. We request patients use an automated blood pressure cuff and home scale to keep logs of their blood pressures, heart rates, and daily weights so that we may review these during our visit and titrate guideline-directed medical therapy. Our patients are able to be remotely evaluated for arrhythmias with event monitors, Apple watches, and KardiaMobile$6 \mathrm{~L}$, which is particularly useful in patients on arrhythmogenic agents such as ibrutinib. These devices could also be used for remote QT interval monitoring. Given the potential for QT prolongation of many established and investigational cancer therapies, remote monitoring would eliminate the need for patients to frequently return to the healthcare setting for routine electrocardiograms [19]. This technology would facilitate monitoring to prevent lifethreatening arrhythmia and enable patients to more easily participate in clinical trials.

While there are definite benefits to telemedicine and increasing access to cardio-oncologists for patients, there are also several limitations for patients and cardio-oncology trainees that must be considered. Patients must have access to a computer or smart phone to participate in telemedicine visits and they must have the technology knowledge to allow them to connect with their providers. Cardio-oncologists must be cognizant of these limitations to avoid marginalizing patients with limited technology knowledge which occurs more commonly in the geriatric population and patients of lower socioeconomic status who may not have access to the technology.

Cardio-oncologists must adapt to these new methods of delivering care and ensure the cardio-oncology trainee is able to participate in these outpatient telemedicine visits. Most trainees do not learn about telemedicine or best practices for this modality of interacting with patients in the way they learn to conduct an outpatient visit in medical school and residency training. Cardiooncologists must therefore set expectations for their trainees and help them to develop best practices. Parikh et al. discuss how to facilitate a telemedicine visit including using a thoughtful "webside" manner to ensure a patient feels connected to his provider, including making eye contact and acknowledging the change in setting from a traditional office visit [20]. There are some aspects of the in-person visit that are important to maintain with the telemedicine visit including that the trainee wear professional attire, maintain a quiet environment for the visit, and adhere to allotted time for visits to be respectful of both the patients' and the providers' time. During these encounters, it is important for the cardio-oncology trainee to be able to review a patient's cardiac and oncologic records, obtain a detailed history from the patient, determine if any additional testing is necessary, and provide recommendations to the patient and the referring provider. These skills can be built upon with successive patient encounters, with the goal of the trainee being able to practice independently by the end of training. Each patient encounter offers a unique learning opportunity 
for the trainee. In our clinic, the trainee is directed to literature relevant to the patient condition to assist with building an evidence-based repository of cardiooncology information. To enable the trainee to learn and develop, it is also imperative that they receive timely and specific feedback about their "webside manner", clinical decision making, and clinical documentation [21]. Providing feedback in the telehealth setting may be difficult due to time and space constraints. Some techniques we employ at MSK to facilitate feedback include the trainee identifying topics and skills they hope to build upon at the beginning of each clinic session, setting aside time for a brief telephone call or video meeting immediately following the clinic session or the next day to allow for reflection and directed feedback, and utilizing e-mail communication to provide timely feedback on each trainee's clinical assessment and documentation.

While it is important to minimize patient exposures during the COVID-19 pandemic, it is equally important to ensure patients who must be seen are able to do so safely. Several groups including the International Cardio Oncology Society (ICOS) and Addison et al. provide guidance as to how cardiooncologists can facilitate appropriate triage of patients, ensuring those at highest risk and those requiring an in-person exam for assessment are able to do so $[17 \bullet \bullet, 22 \bullet]$. When these patients do come for in-person visits, it is essential to minimize risk for disease transmission. At our in-person clinic visits, we follow hospital guidelines, with diligent hand washing, wearing a surgical face mask and protective face shield, social distancing when not performing physical examinations, and ensuring appropriate exam room and equipment cleaning between patients. With adequate PPE and increasing accessibility to COVID-19 vaccination, more visits are being performed in-person if recommended by the cardio-oncologist and agreed to by the patient.

The COVID-19 pandemic has allowed cardio-oncologists and trainees to reassess the mode and frequency for monitoring for potential cardiotoxicity of chemotherapy. Guidelines from the American Society of Clinical Oncology (ASCO) and the European Society of Medical Oncology (ESMO suggest recommendations for use of echocardiography and cardiac biomarkers to monitor patients currently receiving or who have previous exposure to chemotherapies with known cardiac toxicities [23, 24]. Several authors have proposed recommendations for how to adapt cardiac surveillance during the COVID pandemic, but these are not standardized and range from omitting baseline testing in patients with low cardiovascular risk, minimizing imaging in asymptomatic patients, using biomarkers over cardiac imaging, utilizing focused echocardiography rather than complete studies, and using multigated acquisition scans (MUGAs) rather than echocardiography to minimize patient and sonographer exposure $[22 \bullet, 25]$. ICOS has also provided guidance for how to adapt these guidelines during the COVID-19 pandemic, to minimize patient exposure while ensuring adequate cardiac surveillance $[17 \bullet \bullet]$. Using this general framework and the patient's risk tolerance for exposure, the decision on altering testing frequency must be individualized.

\section{Inpatient clinical experience}

The inpatient assessment of the cardio-oncology patient is a critical component for cardio-oncology trainees. The ACC Cardio Oncology Leadership Council 
recommend trainees have exposure to 50 (level II) or 100 (level III) unique patient encounters $[4 \bullet \bullet]$. During the COVID-19 pandemic, these numbers may be difficult to achieve, particularly for trainees who may be redeployed to COVID-19 wards. Due to the number of patients with COVID-19 overwhelming hospitals nationwide, the Accreditation Council for Graduate Medical Education (ACGME) put forth the Three Stages of GME During the Pandemic, with stage 3 allowing programs to declare Pandemic Emergency Status and suspend traditional educational activities for 30 days [26]. Under these regulations, cardiology and oncology fellows could be re-deployed and internal medicine and family medicine residents may miss elective time to serve on general medicine wards and intensive care unit teams. Based upon their training in the care of the critically ill cardiology patient, approximately $38 \%$ of surveyed cardiology fellows reported being re-deployed to care for non-cardiac intensive care unit and floor COVID-19 patients [27]. While this might mean less dedicated time for trainees to pursue cardio-oncology electives or see cardiooncology inpatient consults, caring for COVID-19 patients has multiple similarities to caring for cardio-oncology patients. In the broader sense, cardiooncology requires real-time communication with multiple providers of different subspecialties to make challenging decisions for complicated patients. Similarly, patients hospitalized with COVID-19 tend to be older with more comorbidities and multiple organ systems impacted by the virus. Trainees caring for these patients must quickly review the available and rapidly changing literature and collaborate with multiple consulting teams to make timely treatment decisions. There are several other similarities between COVID-19 and cardio-oncology that allow for trainee education with several examples being risk for QT prolongation, venous thromboembolism (VTE), and cytokine storm. Similar to how tyrosine kinase inhibitors and other chemotherapeutic agents can prolong the QT interval, so too can the proposed treatment regimens for COVID-19 for example hydroxychloroquine, azithromycin, and lopinavirritonavir $[28,29]$. Therefore, an important lesson to trainees caring for these patients is accurate QT interval assessment, measures to mitigate QT prolongation, and when to discontinue therapy to avoid putting patients at risk for ventricular arrhythmia and sudden cardiac death. VTE are commonly seen in patients with cancer and with COVID-19; therefore, both populations provide trainees with experience in diagnosing and treating deep vein thromboses and pulmonary emboli in patients on multiple potentially experimental therapies with possible drug-drug interactions [30, 31]. Lastly, patients with COVID-19 appear to have an exaggerated immune response, a "cytokine storm" that has been compared to the cytokine release syndrome (CRS) seen in patients receiving chimeric antigen receptor $\mathrm{T}$ cells (CAR-T) [32]. Trainees taking care of patients with COVID-19 may gain experience using immunomodulating therapy such as the IL-6 antagonist tocilizumab which is being studied for the treatment of COVID-19 and is currently used to treat CRS in patients receiving CAR-T therapy $[22 \bullet]$.

Cardio-oncology fellows at Memorial Sloan Kettering are integral members of the inpatient cardio-oncology consultative service. During the height of the pandemic in New York, select inpatient consultations were performed via telemedicine, but with decreasing case rates in New York, adequate supply of PPE, and clear guidance from hospital infection control and leadership, inpatient consultations are seen in person with appropriate PPE. In addition to learning 
about traditional cardio-oncology topics, cardio-oncology fellows are further challenged by managing patients with cancer, cardiovascular disease, and COVID-19. At the height of the pandemic, between March and April 2020, 423 MSK patients were diagnosed with COVID-19, 40\% of whom required hospitalization [10]. In these patients, a consult for an elevated troponin may represent demand ischemia from sepsis, an acute coronary syndrome, right ventricular strain from a pulmonary embolism, a check point inhibitor myocarditis, or COVID-19-related myocarditis [33]. The management of these patients requires real-time interdisciplinary discussions to provide rapid lifesaving treatment while simultaneously trying to minimize unnecessary testing and exposing others to the COVID-19 virus. In this setting, the point of care ultrasound (POCUS) allows providers to use a small easily disinfected device to perform a brief limited cardiac ultrasound to assess for significant pericardial disease, left ventricular dysfunction, or right ventricular sequelae of pulmonary embolism while minimizing patient and sonographer exposure [34]. By taking care of these vulnerable high-risk patients, cardio-oncology trainees will be well equipped to serve as independent practicing cardio-oncologists.

\section{Didactics}

Robust didactic sessions are essential to cardio-oncology training. While didactic sessions have traditionally involved cardio-oncologists and trainees meeting together in a conference room, these sessions have transitioned to virtual settings due to social distancing protocols. At MSK, trainees participate in Zoom (San Jose, CA) sessions with cardio-oncology faculty to learn about the core topics of cardio-oncology ranging from anthracycline and trastuzumab cardiotoxicity to clonal hematopoiesis in the oncology patient. Our weekly case conferences, where interesting and challenging cardio-oncology cases are presented, are now held virtually, allowing trainees, faculty, and guest faculty from various disciplines to join even when they may be offsite. Additional imaging teaching sessions, previously held in the echo, CT, nuclear, and MRI reading rooms are now held via virtual platforms, allowing our trainees to gain key competencies in advanced cardiac imaging modalities.

Through virtual platforms, cardio-oncology trainees have access not only to local experts but also to those across the country and around the world. This has taken the form of new programming, such as the International Cardio Oncology Society's weekly webinars and reading with the experts put on by the American Society of Nuclear Cardiology, the Society of Cardiac Magnetic Resonance Imaging, and the Society of Cardiac Computed Tomography [35, 36]. Traditionally, in-person cardio-oncology conferences such as the ACC sponsored "Advancing the Cardiovascular Care of the Cancer Patient", the Memorial Sloan Kettering "Cardio-Oncology Lecture Series", and the ICOS Global CardioOncology Summit have transitioned to virtual platforms. This format is more inclusive for trainees, who may be limited by financial or logistical reasons from attending in-person conferences [37]. Virtual sessions can be recorded which allows for asynchronous learning and may be preferred by adult learners. Lastly, the COVID-19 pandemic has greatly encouraged the use of social media as an educational tool $[38,39]$. Using hashtags such as \#CardioOncology and \#CardioOnc, Twitter users have been able to share new publications, collaborate for 
online journal clubs, network, assist with career development, and learn from each other via "tweetorials" [40,41]. While social media is an exciting, relatively new educational platform, learners must exercise caution as information shared is not subject to the peer review process. With guidance from faculty, the cardiooncology fellows at MSK have been able to develop a presence on Twitter and identify reputable cardio-oncology thought leaders to follow, ensuring a trusted source for cardio-oncology updates. While the use of these learning modalities was sparked by the global pandemic, $73 \%$ of surveyed cardiology fellows have expressed interest in these complimentary online resources being used in combination or in place of traditional learning modalities [27]. Online programming affords enhanced flexibility for adult learners and provides greater access to educational resources previously inaccessible.

Another part of the informal didactic curriculum that is lost due to social distancing protocols is the opportunity for in-person comradery with peers and seniors. The COVID-19 era is laden with uncertainty and despair-a stressful time to be a trainee. Now more than ever, trainee wellness is of utmost importance and cardio-oncology programs must be mindful of this. Trainees should have readily available access to in-person or virtual debriefing sessions with colleagues, program leadership, and mental health professionals to promote resilience and reduce compassion fatigue and burnout. Due to telemedicine, remote learning, and research, trainees may feel isolated. This can be combated by weekly scheduled meetings with trainees and program leadership as well as more informal social events such as virtual "happy hours" and small luncheons. Developing these important mental health skills, mindfulness and resilience during training will position cardio-oncology trainees for success at a time when there are increasing rates of burnout in the field of medicine.

Research is fundamental to any academic training program. Fellows completing level II training are encouraged to either start, contribute to, or complete a research project while level III trainees are suggested to complete a scholarly project leading to a publication $[4 \bullet \bullet]$. Both basic science and clinical research have been impacted by the COVID-19 pandemic. Understanding the immune system response to this virus and potential treatment options is in line with understanding the novel immunotherapy regimens used to target cancer cells that may inadvertently have immune system upregulation with adverse cardiovascular side effects (such as myocarditis with immune checkpoint inhibitors and congestive heart failure with CAR-T cell therapy) [22•]. Clinical researchers must decide whether it is safe to have patients come in person for research visits with potential increased exposure to COVID-19. This may decrease patient participation and impede trial enrollment. The pandemic poses challenges for cardio-oncology research but also provides opportunity for growth and development. Similar to how digital health is becoming part of the outpatient visit, it can just as easily be used to remotely monitor study participants' vitals, ECG, and heart rhythms [42]. Decreased participant enrollment at individual centers may push for more multi-center data registries to pool data and resources, encouraging collaboration, and data-sharing. Digital health, which has taken off during the pandemic, is another key area for investigation [43, 44]. 
Information about this modality of delivering healthcare will be particularly important to cardio-oncology where the number of patients far exceeds the number of sub-specialized providers.

Other forms of clinical research including chart and imaging review may be easier to conduct during the pandemic, and with appropriate access, allow trainees to work remotely to comply with social distancing protocols. It has been suggested that for trainees with limited time, which may be during general cardiology or oncology fellowship or due to redeployment during the pandemic, short-term clinical research projects may be more feasible to see to completion [45•]. MSK cardio-oncology fellows continue to actively engage in several high-impact research projects ranging from amyloid heart disease to clonal hematopoiesis. During the COVID-19 pandemic and with social distancing protocols, fellows have predominantly been involved in retrospective clinical research collaborating with research mentors virtually. Monthly research workin-progress meetings previously held in person are now conducted virtually.

\section{Certification}

Cardio-oncology is still in its infancy with limited opportunities for structured fellowship training or formal certification. ICOS has created a certification exam that trainees may take but is not mandatory at this time. It is recommended that trainees' competency be assessed by their training program on a regular basis using the ACGME core competencies of medical knowledge, patient care, practice learning and improvement, interpersonal and communication skills, and professionalism $[4 \bullet \bullet]$. Based upon the experience of six established cardiooncology programs, Tuzovic et al. recommend fellows be evaluated twice a year using these competencies and that fellows keep a log of patient encounters to review with the program director to ensure adequate exposure to the depth and breadth of cardio-oncology conditions [45•]. One requirement that may be challenging for cardio-oncology trainees to meet during the COVID-19 era is the time and case number requirements for COCATS level II-III training. This is not unique to cardio-oncology. During the pandemic in a survey of cardiology fellows, $69 \%$ expressed concerns about meeting COCATS requirements in time for fellowship graduation due to redeployment, cancelled patient visits, and elective procedures [27]. This raises the question of whether programs should follow traditional time bound educational programming or transition to a competency based, time variable system where trainees are not required to spend a fixed number of months or years in training but instead are able to finish training on a unique timeline once they have demonstrated competency in their chosen field [46]. This will be an important concept to consider in the COVID-19 era and beyond as formalized cardio-oncology training is in its early stages and readily amenable to change as we define best practices.

\section{Conclusions}

The COVID-19 pandemic has impacted all areas of cardio-oncology training. Training programs and trainees have readily adapted to this new environment, learning to provide healthcare through telemedicine, to re-visit standard cardiotoxicity monitoring guidelines, and to apply concepts from cardio-oncology to 
the care of patients with COVID-19. Traditional models of teaching have been set aside to make way for virtual didactic sessions, virtual meetings, and learning via social media. Research has had to shift focus, expand to new remote monitoring techniques, and has a new field of digital cardio-oncology to investigate. These practices have been necessary for the safety of the high-risk cardio-oncology patient population and the providers who care for them. Moving forward, it is likely that many of these practices represent an improvement over the status quo and are here to stay. Therefore, we must embrace the challenges of the COVID-19 era and incorporate these lessons into our training of the next generation of cardio-oncologists.

\section{Declarations}

\section{Conflict of Interest}

Stephanie A. Feldman declares that she has no conflict of interest. Jennifer Liu has received consultant fees from Pfizer, Caption Health, Philips Medical, and for service on the Data and Safety Monitoring Board (DSMB) for Caelum Biosciences, Inc. Richard Steingart declares that he has no conflict of interest. Dipti Gupta declares that she has no conflict of interest.

\section{References and Recommended Reading}

Papers of particular interest, published recently, have been highlighted as:

- Of importance

$\bullet \quad$ Of major importance

1. Siegel RL, Miller KD, Fuchs HE, Jemal A. Cancer Statistics, 2021. CA Cancer J Clin. 2021;71(1):7-33.

2. Lenihan DJ, Hartlage G, DeCara J, Blaes A, Finet JE, Lyon AR, et al. Cardio-oncology training: a proposal from the International Cardioncology Society and Canadian Cardiac Oncology Network for a New Multidisciplinary Specialty. J Card Fail. 2016;22(6):465-71.

3. Johnson MN, Steingart R, Carver J. How to develop a cardio-oncology fellowship. Heart Fail Clin.

2017;13(2):361-6.

4.• Alvarez-Cardona JA, Ray J, Carver J, Zaha V, Cheng R, Yang E, et al. Cardio-oncology education and training: JACC Council Perspectives. J Am Coll Cardiol. 2020;76(19):2267-8.

Key publication delineating the scope of practice, training requirements, and core competencies necessary to become a cardio-oncologist.

5. Johns Hopkins University \& Medicine. Coronavirus Resource Center 2021 [Available from: https:// coronavirus.jhu.edu/. Accessed March 15, 2021

6. CDC Centers for Disease Control and Prevention. How COVID-19 Spreads [Available from: https://www.cdc. gov/coronavirus/2019-ncov/prevent-getting-sick/howcovid-spreads.html. Accessed January 30, 2021
7. Liang W, Guan W, Chen R, Wang W, Li J, Xu K, et al. Cancer patients in SARS-CoV-2 infection: a nationwide analysis in China. Lancet Oncol. 2020;21(3):335-7.

8. Zhou F, Yu T, Du R, Fan G, Liu Y, Liu Z, et al. Clinical course and risk factors for mortality of adult inpatients with COVID-19 in Wuhan, China: a retrospective cohort study. Lancet. 2020;395(10229):1054-62.

9. Ganatra S, Dani SS, Redd R, Rieger-Christ K, Patel R, Parikh R, et al. Outcomes of COVID-19 in patients with a history of cancer and comorbid cardiovascular disease. J Natl Compr Canc Netw. 2020;1-10.

10. Robilotti EV, Babady NE, Mead PA, Rolling T, PerezJohnston R, Bernardes M, et al. Determinants of COVID-19 disease severity in patients with cancer. Nat Med. 2020;26(8):1218-23.

11. Jee J, Foote MB, Lumish M, Stonestrom AJ, Wills B, Narendra V, et al. Chemotherapy and COVID-19 outcomes in patients with cancer. J Clin Oncol. 2020;38(30):3538-46.

12. Bolton $\mathrm{KL}$, Koh Y, Foote MB, Im H, Jee J, Sun CH, et al. Clonal hematopoiesis is associated with risk of severe Covid-19. medRxiv. 2020:2020.11.25.20233163.

13. Shah GL, DeWolf S, Lee YJ, Tamari R, Dahi PB, Lavery JA, et al. Favorable outcomes of COVID-19 in 
recipients of hematopoietic cell transplantation. J Clin Invest. 2020;130(12):6656-67.

14. Polack FP, Thomas SJ, Kitchin N, Absalon J, Gurtman A, Lockhart S, et al. Safety and efficacy of the BNT162b2 mRNA Covid-19 vaccine. N Engl J Med. 2020;383(27):2603-15.

15. Baden LR, El Sahly HM, Essink B, Kotloff K, Frey S, Novak R, et al. Efficacy and Safety of the mRNA-1273 SARS-CoV-2 Vaccine. N Engl J Med. 2020.

16. CDC Centers for Disease Control and Prevention. When vaccine is limited, who should get vaccinated first? [Available from: https://www.cdc.gov/ coronavirus/2019-ncov/vaccines/recommendations. html. Accessed January 30, 2021

17.• Lenihan D, Carver J, Porter C, Liu JE, Dent S, Thavendiranathan $\mathrm{P}$, et al. Cardio-oncology care in the era of the coronavirus disease 2019 (COVID-19) pandemic: An International Cardio-Oncology Society (ICOS) statement. CA Cancer J Clin. 2020;70(6):480-50.

Key paper outlining the impact of COVID-19 on cardiooncology with specific recommendations for how to adjust cardio-oncology practices to optimize patient safety and cardiovascular and oncologic outcomes during the pandemic.

18. Brown S-A, Rhee J-W, Guha A, Rao VU. Innovation in precision cardio-oncology during the coronavirus pandemic and into a post-pandemic world. Front Cardiovasc Med. 2020;7:145.

19. Castelletti S, Dagradi F, Goulene K, Danza AI, Baldi E, Stramba-Badiale $\mathrm{M}$, et al. A wearable remote monitoring system for the identification of subjects with a prolonged QT interval or at risk for drug-induced long QT syndrome. Int J Cardiol. 2018;266:89-94.

20. Parikh A, Kumar AA, Jahangir E. Cardio-oncology care in the time of COVID-19 and the role of Telehealth. JACC: CardioOncology. 2020;2(2):356-8.

21. Ramani S, Krackov SK. Twelve tips for giving feedback effectively in the clinical environment. Med Teach. 2012;34(10):787-91.

22. Addison D, Campbell CM, Guha A, Ghosh AK, Dent $\mathrm{SF}$, Jneid H. Cardio-oncology in the era of the COVID19 pandemic and beyond. J Am Heart Assoc. 2020;9(19):e01778.

Important paper describing the impact of COVID-19 on all aspects of cardio-oncology practice with specific recommendations for how to adapt during the pandemic and beyond.

23. Armenian SH, Lacchetti C, Barac A, Carver J, Constine LS, Denduluri N, et al. Prevention and monitoring of cardiac dysfunction in survivors of adult cancers: American Society of Clinical Oncology Clinical Practice Guideline. J Clin Oncol. 2017;35(8):893-911.

24. Curigliano G, Lenihan D, Fradley M, Ganatra S, Barac A, Blaes A, et al. Management of cardiac disease in cancer patients throughout oncological treatment: ESMO consensus recommendations. Ann Oncol. 2020;31(2):171-90.

25. Farmakis D, Keramida K, Filippatos G. Cardiooncology services during the COVID-19 pandemic: practical considerations and challenges. Eur J Heart Fail. 2020;22(6):929-32.

26. Accreditation Council for Graduate Medical Education. Three stages of GME during the COVID-19 pandemic [Available from: https://acgme.org/COVID-19/ThreeStages-of-GME-During-the-COVID-19-Pandemic. Accessed January 20, 2021

27. Rao P, Diamond J, Korjian S, Martin L, Varghese M, Serfas JD, et al. The impact of the COVID-19 pandemic on cardiovascular fellows-in-training: a national survey. J Am Coll Cardiol. 2020;76(7):871-5.

28. Chandrasekhar S, Fradley MG. QT interval prolongation associated with cytotoxic and targeted cancer therapeutics. Curr Treat Options in Oncol. 2019;20(7):55.

29. Jankelson L, Karam G, Becker ML, Chinitz LA, Tsai MC. QT prolongation, torsades de pointes, and sudden death with short courses of chloroquine or hydroxychloroquine as used in COVID-19: A systematic review. Heart Rhythm. 2020;17(9):1472-9.

30. Cohen AT, Katholing A, Rietbrock S, Bamber L, Martinez C. Epidemiology of first and recurrent venous thromboembolism in patients with active cancer. A population-based cohort study. Thromb Haemost. 2017;117(1):57-65.

31. Middeldorp S, Coppens $M$, van Haaps TF, Foppen M, Vlaar AP, Müller MCA, et al. Incidence of venous thromboembolism in hospitalized patients with COVID-19. J Thromb Haemost. 2020;18(8):19952002.

32. Siddiqi HK, Neilan TG. COVID-19, Immuno-oncology and cardiovascular disease: viewpoint from the intersection. J Cardiovasc Transl Res. 2020;13(3):347-8.

33. Mahmood SS, Fradley MG, Cohen JV, Nohria A, Reynolds KL, Heinzerling LM, et al. Myocarditis in patients treated with immune checkpoint inhibitors. J Am Coll Cardiol. 2018;71(16):1755-64.

34. Baldassarre LA, Yang EH, Cheng RK, DeCara JM, Dent $\mathrm{S}$, Liu JE, et al. Cardiovascular care of the oncology patient during COVID-19: an expert consensus document from the ACC Cardio-Oncology and Imaging Councils. J Natl Cancer Inst. 2020.

35. Berookhim J, Correa A, Tamis-Holland JE. Notes from the eye of the storm. J Am Coll Cardiol. 2020;76(2):218-20.

36. Senapati A, Khan N, Chebrolu LB. Impact of social media and virtual learning on cardiology during the COVID-19 pandemic era and beyond. Methodist Debakey Cardiovasc J. 2020;16(3):e1-7.

37. Narula N, Singh HS. Cardiology practice and training post-COVID-19. J Am Coll Cardiol. 2020;76(4):4769.

38. Jaffe RC, O'Glasser AY, Brooks M, Chapman M, Breu AC, Wray CM. Your @Attending Will \#Tweet You Now: Using Twitter in Medical Education. Acad Med. 2020;95(10):1618.

39. Coleman CG, Law KL, Spicer JO. \#EducationInTheTimeOfCOVID: Leveraging social media to teach during 
the COVID-19 pandemic pandemonium. Med Educ. 2020;54(9):852-3.

40. Brown S-A, Daly RP, Duma N, Yang EH, Pemmaraju N, Parwani $\mathrm{P}$, et al. Leveraging Social Media for CardioOncology. Curr Treat Options in Oncol. 2020;21(10):83.

41. Parwani P, Choi AD, Lopez-Mattei J, Raza S, Chen T, Narang A, et al. Understanding social media: opportunities for cardiovascular medicine. J Am Coll Cardiol. 2019;73(9):1089-93.

42. Sharma A, Harrington RA, McClellan MB, Turakhia MP, Eapen ZJ, Steinhubl S, et al. Using digital health technology to better generate evidence and deliver evidence-based care. J Am Coll Cardiol. 2018;71(23):2680-90

43. Jandoo T. WHO guidance for digital health: what it means for researchers. Digit Health. 2020;6:2055207619898984.

44. Gaba P, Bhatt DL. The COVID-19 pandemic: a catalyst to improve clinical trials. Nat Rev Cardiol. 2020;17(11):673-5.
45.• Tuzovic M, Brown S-A, Yang EH, West BH, Bassi NS, Park S, et al. Implementation of cardio-oncology training for cardiology fellows. JACC: CardioOncology. 2020;2(5):795-.

Important publication outlining experiences in developing cardio-oncology programs, keys for success, and potential limitations that need to be addressed.

46. Lucey CR, Johnston SC. The transformational effects of COVID-19 on medical education. JAMA. 2020.

\section{Publisher's note}

Springer Nature remains neutral with regard to jurisdictional claims in published maps and institutional affiliations. 